\title{
Models of M 67
}

\author{
Jarrod R. Hurley \\ Centre for Astrophysics and Supercomputing, Swinburne University of Technology, \\ PO Box 218, Hawthorn, VIC 3122, Australia \\ email: jhurley@astro.swin.edu.au
}

\begin{abstract}
The old open cluster M 67 is an ideal test case for current star cluster evolution models because of its dynamically evolved structure and rich stellar populations that show clear signs of interaction between stellar, binary and cluster evolution. Here we discuss a direct N-body model of M 67 . This model of 12,000 single stars and 12,000 binaries is evolved from zero-age and takes full account of cluster dynamics as well as stellar and binary evolution. At an age of 4 Gyr the model cluster matches the mass and structure of M 67 as constrained by observations. We discuss the role of the primordial binary population and the cluster environment in shaping the nature of the stellar populations of M 67, with a focus on X-ray binaries and blue stragglers.
\end{abstract}

Keywords. stellar dynamics, methods: n-body simulations, binaries: close, blue stragglers

Despite advances in computing power made over the last decade or so, the globular clusters of our Galaxy remain out of reach of the direct $N$-body method. However, old open clusters such as M 67 and NGC 188 with ages in the range 4 - 8 Gyr offer dynamically evolved systems where the number, $N$, of stars required in the starting model is less than 100000 and within current limitations (which also depend on the primordial binary fraction). A large number (29) of blue stragglers (BSs) are observed in M67 and there are a number of indicators that the cluster environment, in addition to close binary evolution, has played a role in shaping this population. These include: $(i)$ the BSs are concentrated towards the centre of M 67; (ii) BSs are found in eccentric binaries (with an orbital period of $P \sim 4 \mathrm{~d}$ in one case: see Latham, these proceedings); and, (iii) the ratio of BSs to main-sequence stars is excessive for an open cluster and greater than can be produced by standard binary population synthesis (see Hurley et al. 2001). An $N$-body model of M 67 was presented by Hurley et al. (2001) and showed that binary evolution in combination with 3- and 4-body interactions can create the variety of BSs and BS-binaries observed. However, this model was semi-direct in that the dynamical evolution was not modelled directly for the first $2.5 \mathrm{Gyr}$ of the cluster lifetime. Thus it was not ideal.

A preferred model of M 67 was presented by Hurley et al. (2005). This started with 12000 single stars and 12000 binaries. Stellar masses were chosen from the initial mass function of Kroupa et al. (1993) between the limits of 0.1-50 $\mathrm{M}_{\odot}$ to give a total mass of $18700 \mathrm{M}_{\odot}$. The cluster was placed on a circular orbit at $8 \mathrm{kpc}$ from the Galactic centre - the time averaged semimajor axis for M 67 which has a slightly eccentric orbit - with an orbital speed of $220 \mathrm{~km} \mathrm{~s}^{-1}$. This gave an initial tidal radius of $32 \mathrm{pc}$. A Plummer density profile was assumed for the starting model with the stars in virial equilibrium. Solar metallicity was assumed.

The model was evolved to an age of 5 Gyr using NBODY4 (Aarseth 1999) - a Hermite integration code utilising GRAPE-6 hardware with stellar and binary evolution included as described in Hurley et al. (2001). At an age of $4 \mathrm{Gyr}$ the model cluster has a mass of $\sim 2000 \mathrm{M}_{\odot}$ within a tidal radius of $15 \mathrm{pc}$, a half-mass radius of $2.7 \mathrm{pc}$ and a binary frequency of $50 \%$. This provides an excellent match to the properties of M 67 (see (Fan 


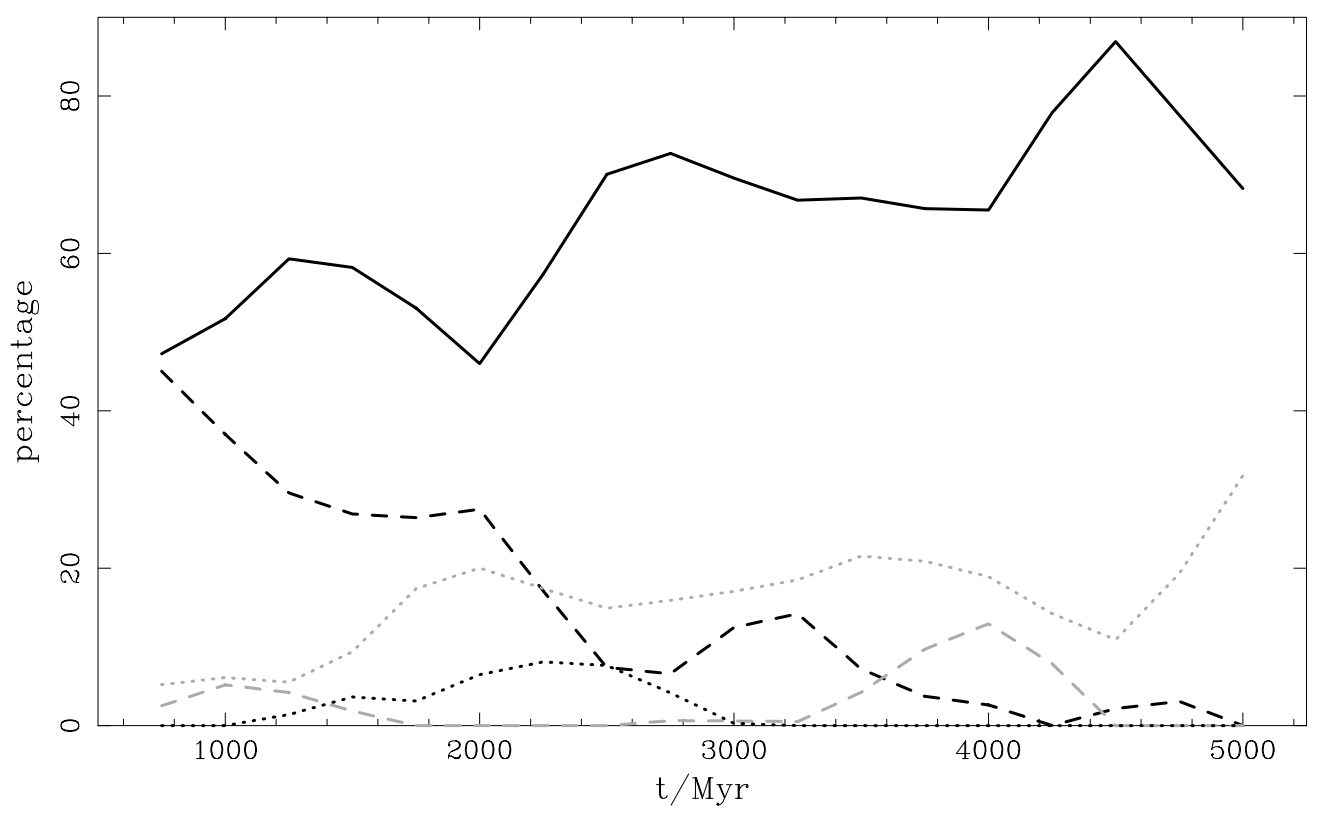

Figure 1. Blue straggler configurations as a function of time for the M $67 \mathrm{~N}$-body model. Shown are single BSs (solid line) and BSs in binaries with $P<1000 \mathrm{~d}$ (dashed lines) or longer periods (dotted lines - bold/grey indicates circular/eccentric orbits).

et al. 1996, for example). We note that the core binary fraction rises from 0.5 to 0.8 during the simulation and is thus not depleted even if neglected soft binaries are counted.

The model at 4 Gyr contains 20 BSs with 8 in eccentric binaries. The half-mass radius of the BSs is $1.1 \mathrm{pc}$. Figure 1 summarizes the respective BS configurations as the model evolves - single BSs dominate but the proportion of BSs in eccentric binaries grows with time. Indications for M 67 are that $50 \%$ of the BSs are single with $20 \%$ in short-period binaries (all eccentric) and the remainder in long-period binaries $(\sim 20 \%$ eccentric, $\sim 10 \%$ circular). The 12000 primordial binaries in the model had periods drawn from a flat distribution of $\log (P)$. Evolving these with a binary evolution algorithm (no dynamics) predicts 25 BSs at 4 Gyr with $75 \%$ single and $25 \%$ in circular binaries. So the cluster environment creates a more realistic configuration spread but also destroys potential BSs via hardening of close binaries. Repeating the $N$-body model with initial periods drawn from the distribution of Kroupa (1995) - which gives a $50 \%$ reduction in short-period binaries - produces only one BS at $4 \mathrm{Gyr}$. This also leads to a reduced number of X-ray active BY Draconis binaries (see Hurley et al. 2005 for a full explanation and references).

\section{Acknowledgements}

JRH would like to thank the IAU for assistance in the form of a travel grant.

\section{References}

Aarseth, S. J. 1999, PASP, 111, 1333

Fan, X., Burstein, D., Chen, J.-S., et al. 1996, AJ, 112, 628

Hurley, J. R., Tout, C. A., Aarseth, S. J., \& Pols, O. R. 2001, MNRAS, 323, 630

Hurley, J. R., Pols, O. R., Aarseth, S. J., \& Tout, C. A. 2005, MNRAS, 363, 293

Kroupa, P. 1995, MNRAS, 277, 1507

Kroupa, P., Tout, C. A., \& Gilmore, G. 1993, MNRAS, 262, 545 